\title{
Diacronie
}

Studi di Storia Contemporanea

$\mathrm{N}^{\circ} 36,4 \mid 2018$

Viaggi e turismo nell'Europa del Novecento

\section{Le voyage à Alger dans les guides touristiques français au $\mathrm{XX}^{\mathrm{e}}$ siècle}

\section{Sihem Bella}

\section{Q OpenEdition}

1 Journals

\section{Édition électronique}

URL : https://journals.openedition.org/diacronie/10531

DOI : 10.4000/diacronie. 10531

ISSN : 2038-0925

Éditeur

Association culturelle Diacronie

Référence électronique

Sihem Bella, «Le voyage à Alger dans les guides touristiques français au XXe siècle », Diacronie [En ligne], $N^{\circ}$ 36, 4 | 2018, document 10, mis en ligne le 29 décembre 2018, consulté le 17 février 2022. URL : http://journals.openedition.org/diacronie/10531; DOI : https://doi.org/10.4000/diacronie.10531 


\section{Diacronie}

Studi di Storia Contemporanea

36, 4/2018

Viaggi e turismo nell'Europa del Novecento

\section{Le voyage à Alger dans les guides touristiques français au $\mathrm{XX}^{\mathrm{e}}$ siècle}

\section{Sihem BELLA}

Per citare questo articolo:

BELLA, Sihem, «Le voyage à Alger dans les guides touristiques français au XX ${ }^{\mathrm{e}}$ siècle», Diacronie. Studi di Storia Contemporanea : Viaggi e turismo nell'Europa del Novecento, 36, 4/2018, 29/12/2018,

URL: < http://www.studistorici.com/2018/12/29/bella_numero_36/ >

Diacronie Studi di Storia Contemporanea $\rightarrow$ http://www.diacronie.it

Rivista storica online. Uscita trimestrale.

redazione.diacronie@hotmail.it

Comitato di direzione: Naor Ben-Yehoyada - João Fábio Bertonha - Christopher Denis-Delacour - Maximiliano Fuentes Codera Anders Granås Kjøstvedt - John Paul Newman - Deborah Paci - Niccolò Pianciola - Spyridon Ploumidis - Wilko Graf Von Hardenberg

Comitato di redazione: Jacopo Bassi - Luca Bufarale - Gianluca Canè - Luca G. Manenti - Fausto Pietrancosta - Alessandro Salvador - Matteo Tomasoni - Luca Zuccolo

Diritti: gli articoli di Diacronie. Studi di Storia Contemporanea sono pubblicati sotto licenza Creative Commons 3.0. Possono essere riprodotti e modificati a patto di indicare eventuali modifiche dei contenuti, di riconoscere la paternità dell'opera e di condividerla allo stesso modo. La citazione di estratti è comunque sempre autorizzata, nei limiti previsti dalla legge. 


\title{
10/ Le voyage à Alger dans les guides touristiques français au $\mathrm{XX}^{\mathrm{e}}$ siècle
}

\author{
Sihem BELLA
}

Comment les guides touristiques permettent-ils de s'intéresser à la rencontre entre les touristes français et la ville d'Alger, joyau de leur empire colonial ? Ils constituent une source riche pour l'histoire culturelle de l'Algérie coloniale. Ils nous renseignent sur les motifs du voyage, les pratiques touristiques et sur la charge symbolique qui pèse sur ce périple. Les guides cristallisent les perceptions métropolitaines d'Alger, ainsi que des représentations éminemment politiques : ils participent à la construction d'un imaginaire national français autour de la ville. Les guides délivrent un discours cohérent, une grille de lecture de la ville pour l'historien, et contribuent à la compréhension historique d'une expérience vécue par des millions de Français.

\section{Introduction : le guide touristique, une source pour l'histoire culturelle de l'Algérie coloniale}

«Conduire ou rendre possible la conduite dans un espace inconnu ou insuffisamment connu» ${ }^{1}$ : le guide touristique peut se définir ainsi. Produit de la révolution de l'imprimé et du développement du tourisme de masse, le genre est l'un des représentants les plus emblématiques de la littérature de voyage. Le guide est périssable, il est donc un objet inscrit dans son époque : c'est précisément ce qui en fait une source intéressante pour l'historien. Son aspect pratique est essentiel : le guide doit guider. Il indique au touriste comment se déplacer vers et à travers la ville, où manger, où dormir, quoi voir et quoi faire. L'ouvrage adopte toujours un petit format : inoctavo au maximum, le plus souvent in-seize. L'ambition est d'offrir une image globale et complète de la ville, et de délivrer un discours normatif sur le territoire concerné. Le guide est en quelque sorte la version standardisée du récit de voyage, mais une version déclassée : il s'agit

${ }^{1}$ Avant-propos, in CHABAUD, Gilles, COHEN, Evelyne, COQUERY, Natacha, PENEZ, Jérôme, (textes réunis par), Les Guides imprimés du XVI $I^{e}$ au XX $X^{e}$ siècle. Villes, paysages, voyages, d'après un colloque de 1998, Université Paris VII, Paris, Belin, 2000, p. 9. 
d'une marchandise, périssable de surcroît, dont le prix figure souvent en évidence sur la couverture. Les guides représentent une source abondante pour l'historien de l'Europe du XX siècle, notamment en raison de la pérennité des grandes collections. Le guide permet de donner à l'espace l'unité dont le chercheur manque parfois pour l'aborder. Il le lisse, le met en ordre, le hiérarchise, le rend lisible par l'historien. L'essor des guides comme sources historiques s'inscrit dans le contexte beaucoup plus large du développement de l'histoire culturelle. Les guides sont en effet des sources livresques privilégiées pour étudier les représentations et les imaginaires autour de l'espace urbain.

La recherche sur laquelle porte l'article est basée ${ }^{2}$ sur les sources imprimées que sont les guides touristiques d'Alger. L'étude s'étend sur le temps long du $\mathrm{XX}^{\mathrm{e}}$ siècle colonial français, jusqu'à l'indépendance en 1962, ce qui représente une trentaine de guides. Alger est alors la capitale de l'Algérie française. "Indigène » et européenne, place militaire et comptoir colonial, Alger est à la fois une grande métropole moderne et une médina arabe. Premier bastion de la France en Afrique, Alger est en 1962 la quatrième ville française après Paris, Lyon et Marseille. La mise en valeur du territoire algérien, l'avènement des classes moyennes en France, les progrès des transports et de la communication, la prise en compte du climat comme élément de qualité de vie et la mode de l'hivernage amenèrent, au fil de la période, de plus en plus de voyageurs à Alger. Seuls les guides édités en France ont été retenus, puisqu'il s'agit des rapports des Français à leur empire colonial.

Cet article a pour ambition d'interroger la signification du voyage à Alger pour les Français à l'époque coloniale. Les guides contiennent des représentations éminemment politiques qui rendent la destination touristique prestigieuse, et qui contribuent à constituer un véritable imaginaire national autour de la ville coloniale. Le voyage à Alger peut même prendre un sens patriotique. Nous verrons dans quelle mesure les guides de voyage reflètent, diffusent et légitiment une façon de voyager à Alger. Les représentations qu'ils donnent à voir font système avec l'ensemble des représentations que la société a de son territoire. Le guide de voyage pose en effet la question de l'image de l'autre qu'il véhicule et du regard de celui qui le porte ; la situation coloniale ajoute un paramètre supplémentaire, dans le cas d'Alger. L'idée est d'étudier les perceptions métropolitaines d'Alger; il s'agit d'une histoire européenne de l'Algérie, vue du dehors.

\footnotetext{
${ }^{2}$ D'après un travail de recherche mené à Sciences Po (Paris) sous la direction de Jean-François Sirinelli, à qui nous adressons ici nos remerciements.
} 


\section{Le guide touristique d'Alger : stratégies de formes et de contenus pour raconter la ville coloniale}

\subsection{Essai de typologie des guides touristiques d'Alger}

C'est l'objet que constitue le guide d'Alger qui retiendra d'abord notre attention. Les formes et les contenus que le guide d'Alger adopte au cours de la période sont le résultat de choix, de stratégies de la part de ses concepteurs. L'évolution d'un genre éditorial sur plusieurs décennies n'est pas chose aisée à retracer. La formalisation du guide touristique ne concerne pas que les guides d'Alger, mais le genre tout entier. Celui-ci connaît un essor éditorial important au XIX siècle, dans toute l'édition européenne (principalement allemande, anglaise et française). Le guide touristique vient indubitablement du récit de voyage, comme en témoigne le guide Promenades à Alger d'Alfred-Samuel Gubb ${ }^{3}$. Dans un style romancé, l'auteur accorde le fil de sa marche au fil de sa plume. Outre ces "guides d'auteurs" apparentés aux récits de voyage, d'importantes collections de guides se constituent au fil du XIX ${ }^{e}$ siècle. Ces collections constituent l'écrasante majorité de notre corpus : ceux des maisons d'édition Hachette et Michelin principalement. Hachette édite le célèbre Guide-Joanne, remplacé en 1919 par le Guide bleu : le premier Guide bleu Algérie Tunisie est publié en $1923^{4}$. Dès 1855 , les guides d'Algérie sont compris dans la souscollection des guides de France: le fait est révélateur, et permet d'emblée de tirer des interprétations au sujet des constructions régionales, nationales voire impériales dont les guides touristiques sont les reflets. Après Hachette, la deuxième maison d'édition la plus importante de notre corpus est Michelin. Le fabricant de pneumatiques est en effet l'un des éditeurs touristiques les plus prospères au début du XX $\mathrm{XX}^{\mathrm{e}}$ siècle ${ }^{5}$. Le premier guide de la maison Michelin paraît en 1900, et le premier guide Algérie-Tunisie en $1905^{6}$. L'esprit des guides Michelin s'oppose en de nombreux points à celui des guides de la maison Hachette : la typographie est plus large et plus aérée que dans les derniers, et le vocabulaire se veut plus simple et plus accessible. Le public visé n'est pas le même: les guides Michelin sont pour des raisons évidentes très centrés sur l'automobile.

Outre les grandes collections de Michelin et de Hachette, d'autres collections de guides apparaissent au fil de la période, comme les guides Dunlop, les guides Portier ou encore les guides Conty. La moitié seulement des guides retenus pour cette étude font mention d'auteurs. Les

\footnotetext{
3 GUBB, Alfred-Samuel, Promenades à Alger. Excursions en Algérie. Itinéraires et plans, Alger, éditions Jules Carbonel, 1921.

4 RICARD, Prosper, DALBANNE, Jean, DRAPPIER, Léon, Guide bleu Algérie Tunisie, Paris, Hachette, 1923.

5 Voir la thèse de: FRANCON, Marc, Le Guide vert Michelin. L'invention du tourisme culturel populaire, Paris, Economica, 2001.

${ }^{6}$ Guide Michelin pour l'Algérie et la Tunisie, Tours, Marne, 1905.
} 
éditions Hachette font appel à des spécialistes ou des universitaires reconnus, lesquels sont cités afin d'ajouter quelque prestige aux guides. En revanche, certains éditeurs comme Michelin font le choix manifeste de l'anonymat des guides : il s'agit probablement de conférer un caractère simple, impersonnel à l'ouvrage, de le rendre accessible aux lecteurs. Autre collection représentée dans notre corpus, les guides Dunlop, qui suivent la niche éditoriale des guides automobiles. En 1923 paraît le guide de Jean du Taillis, Le Tourisme automobile en Algérie-Tunisie ${ }^{7}$. Bien qu'intégré à une collection, le guide est signé : exceptés les Guides-Joanne surtout associés à Louis Piesse, il s'agit du seul cas de " guide d'auteur » intégrant une collection. Le but est affiché : le guide Dunlop est certes un guide automobile, mais pour lecteurs cultivés. Un soin particulier est accordé à la couverture, et le papier est de bonne qualité : sur la page de garde est indiqué qu' «il a été tiré de cet ouvrage 100 exemplaires numérotés sur papier de luxe ». Dans " l'avertissement », le rival Michelin est expressément attaqué : « les uns et les autres usurpent le qualificatif de guides », et se cantonnent à une "froide énumération des villes " ${ }^{8}$. D'autres collections se succèdent, sans égaler par leur succès les guides Hachette et Michelin : les guides Portier du nom de l'éditeur, les guides Conty édités par Chaix, les guides Alger en poche ou encore les Alger-guide édités chez Heintz. Ces guides sont essentiellement pratiques et relayent par exemple les horaires de traversées des bateaux entre Marseille et Alger ou les adresses utiles d'Alger. Un dernier ensemble de guides, qui peut difficilement correspondre à la dénomination de « collection » sont les brochures émanant des syndicats d'initiative au tourisme et autres comités d'hivernage, dont la date et l'éditeur apparaissent assez peu clairement. Les collections représentées dans notre corpus se distinguent ainsi par le type de lecteurs auxquels ils sont destinés, par leur usage et leur fonction - qui se rapportent, la plupart du temps, au prestige de la collection. Il est malheureusement difficile de quantifier à la fois les tirages et les ventes des collections rivales ; la confidentialité de ces informations était savamment entretenue.

Qui sont les lecteurs-touristes des guides d'Alger? L'aporie est fréquente en histoire culturelle: il n'est pas possible d'avoir une connaissance directe des lecteurs en disposant uniquement des livres qu'ils ont lus. L'historien doit alors se contenter de réfléchir à la question du public visé par les guides touristiques en explorant d'autres pistes. La publicité en est une : elle peut permettre l'identification d'un public visé. Dans les guides, la promotion de compagnies de transports, d'hôtels ou encore de restaurants est fréquente. Certains guides paraissent sont plus genrés que d'autres: dans ceux qui semblent destinés aux femmes, en plus de choix formels

\footnotetext{
${ }^{7}$ TAILLIS, Jean (du), Le Tourisme automobile en Algérie-Tunisie. Guide Dunlop, Paris, éditions des guides de Tourisme automobile, 1923, p. 57.

8 Ibidem. D'après Arnaud Berthonnet, « cette publication fait suite à un ouvrage paru en 1922 sur le tourisme automobile au Maroc, dont le succès a été considérable puisque les 5000 exemplaires de la première édition ont été enlevés en quelques mois ». BERTHONNET, Arnaud, "Le tourisme en Algérie (de 1880 aux années 1940) : une histoire à écrire ", in KAZDAGHLI Habib, ZYTNICKI Colette, BOUMEGGOUTI Driss, VALERO Alet (dir.), Tourisme: Pour une histoire du tourisme au Maghreb, XIX ${ }^{\mathrm{e}}-X^{e}$ siècles, 15, 2006, pp. 1-19.
} 
comme les couleurs et les illustrations, les publicités sont orientées. A titre d'exemple, le LivretGuide illustré daté de 1908 regorge d'annonces pour les salons de beauté algérois, les bains maures ou encore les grands magasins ${ }^{9}$. Enfin, de manière générale, les guides sont destinés à des lecteurs-touristes privilégiés, d'un point de vue à la fois économique et culturel : d'après l'estimation de l'historienne Hélène Morlier, un Guide-Joanne coûterait aujourd'hui l'équivalent d'une trentaine d'euros ${ }^{10}$.

\subsection{L'ambition d'un ouvrage total : le guide d'Alger ou tout ce qu'il faut voir et savoir sur cette ville}

L'ambition d'exhaustivité est au cœur d'un genre comme le guide touristique. Le travail des rédacteurs consiste à donner l'impression aux voyageurs que tout ce qui doit être vu ou su au sujet d'Alger se trouve dans les pages du guide. Les cartes et les plans sont des appendices essentiels dans les guides de voyage, pour des raisons évidentes : le touriste les regarde pour se repérer dans la région et dans la ville. La carte est le « simulacre qui permet de s'approprier dans un seul regard la totalité» ${ }^{11}$. Ce qui n'empêche pas que l'usage des cartes dans les guides révèle une certaine subjectivité: une proportion importante de guides et de plans attachés aux guides du corpus trahit une vision éminemment franco-centrée de la ville coloniale. Faisant fi de l'orientation basée sur les points cardinaux, l'orientation est choisie de telle façon que la ville paraisse tournée vers la France; Alger semble ainsi s'offrir au touriste français arrivant par la mer. Les cartes et les plans participent à bien des égards à une culture visuelle des empires : il s'agit d'éduquer, voire de formater un regard sur le monde colonial. Ces représentations cartographiques ont une dimension performative évidente. Dans la plupart des guides, le plan joint présente la mer Méditerranée sur la partie inférieure de la carte. La mer Méditerranée est saisie « à l'envers », du point de vue français.

\footnotetext{
${ }^{9}$ Livret-Guide illustré publié par le Comité d'hivernage algérien, Alger, Aux bureaux du comité, 1908.

${ }^{10}$ MORLIER, Hélène, Les Guides-Joanne. Genèse des Guides-Bleus, Paris, Les Sentiers débattus, 2007, p. 88.

Pour Hélène Morlier, 5 FF équivaudrait à environ 15 euros en 1901. Les guides d'Algérie étaient vendus 12FF en moyenne.

${ }_{11}^{11}$ VERDIER, Nicolas, «Les formes du voyage : cartes et espaces des guides de voyage », in In Situ, 15, 1/2011, pp. 1-18, URL <http://insitu.revues.org/573> [consulté le 11 février 2016]
} 


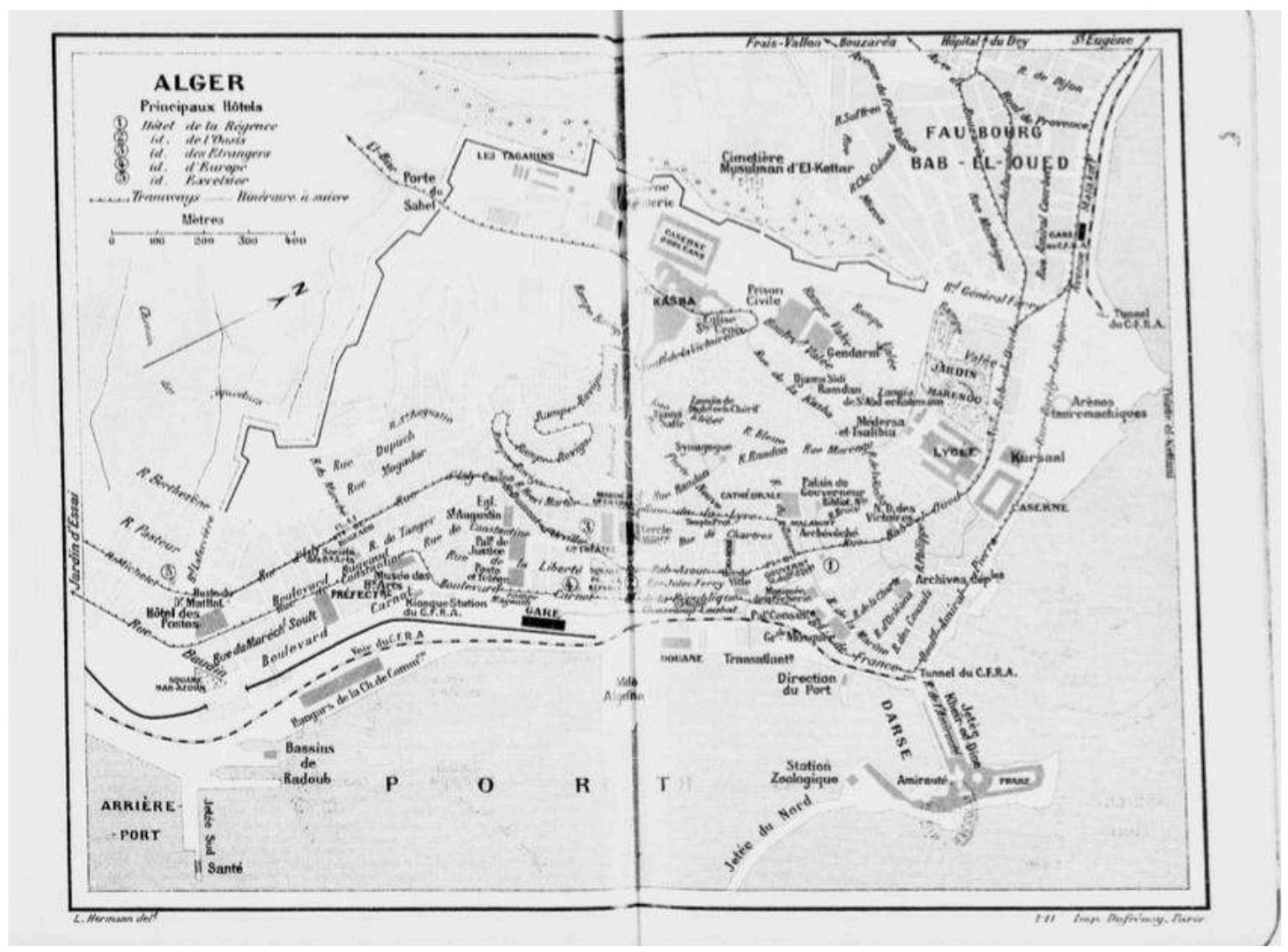

Figure 1. Plan d'Alger.

[Source : JACQUETON, Gilbert, GSELL, Stéphane, Guide-Joanne Algérie Tunisie, Paris, Gallimard, 1911].

La cartographie, est plus largement la géographie, participe du processus de colonisation et de l'exercice du pouvoir colonial. La géographie est souvent considérée par les spécialistes comme la science coloniale par excellence; selon Edward Saïd, l'impérialisme est "acte de violence géographique, par lequel la quasi-totalité de l'espace mondial est explorée, cartographiée et finalement annexée ${ }^{12} »$.

Comme les cartes, les illustrations, photographies et autres gravures sont prépondérants dans des ouvrages aussi soumis aux lois commerciales que les guides : ils doivent plaire. Au fil des décennies, le noir et blanc fait place à la couleur. Les guides Michelin valorisent l'illustration, en cohérence avec leur ligne éditoriale : il y a une nette volonté de rendre le guide accessible, de vulgariser les connaissances qui y sont contenues.

12 SAID, Edward, Culture et impérialisme, Paris, Fayard - Le Monde Diplomatique, 2000 [1 1 ère édition: Culture and Imperialism, New York, Knopf, 1993], p. 320. 
Dans l'édition 1930 du Guide Michelin Maroc Algérie Tunisie, la couverture est très orientalisante : le « bonhomme Michelin » s'affiche sur un chameau en habit traditionnel « indigène ».

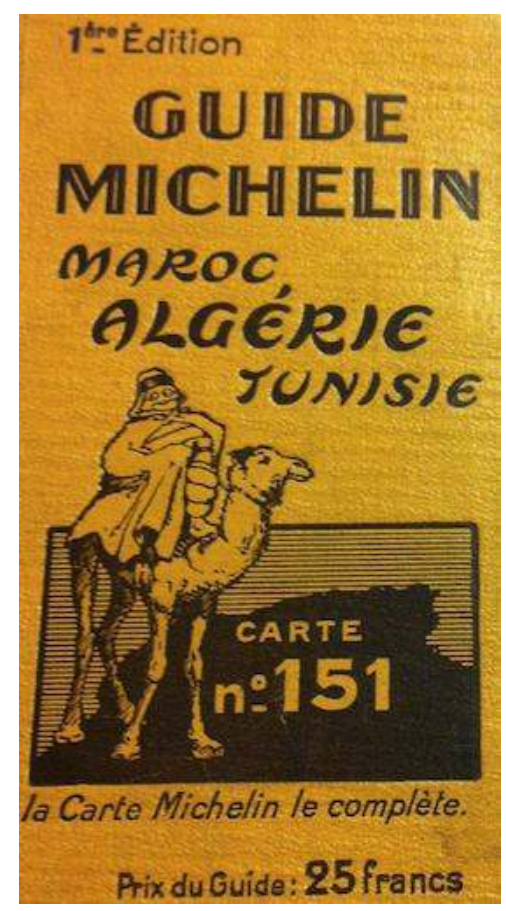

Figure 2. Bibendum « indigène ».

[Source : Guide Michelin Maroc, Algérie, Tunisie, Paris, Michelin, 1927].

La photographie est le type d'illustration le plus répandu dans les guides. La vue d'Alger depuis le bateau est un grand classique. Il correspond à la première vision qu'ont les voyageurs à leur arrivée en bateau. Les images de l'Alger coloniale montrent la facette la plus souvent célébrée dans les guides : Alger comme ville française.

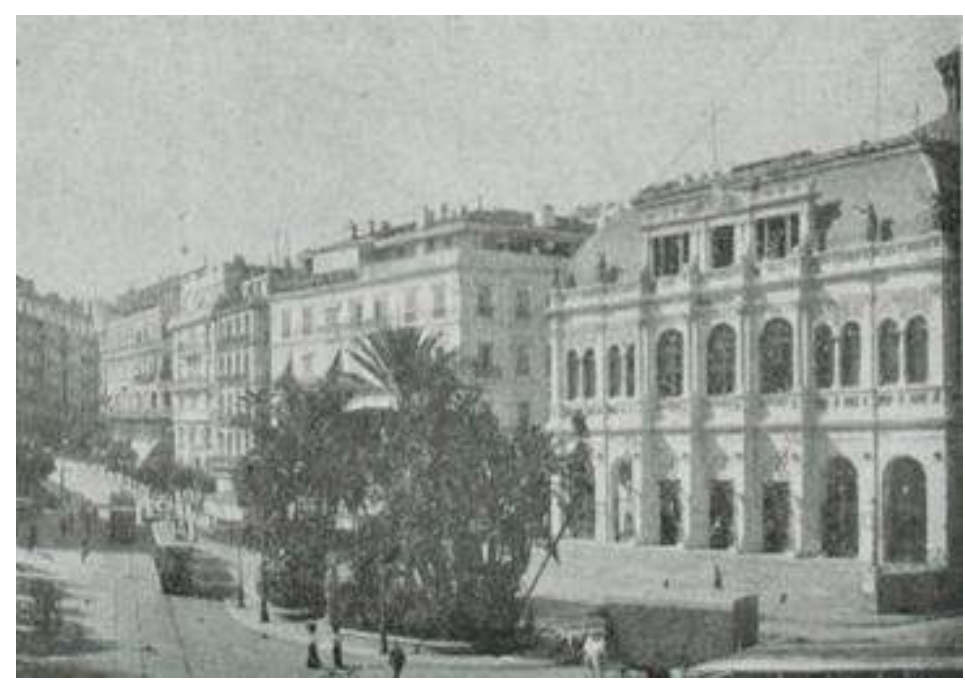

Figure 3. Photographie du théâtre d'Alger

[Source : Livret-guide illustré, publié par le Syndicat d'initiative de Tourisme (ancien Comité d'hivernage algérien), Alger, Jean Dorchies, 1939]. 
Si les guides diffusent une iconographie stéréotypée, leurs auteurs ne l'inventent pas. Ils ne font que confirmer et donner de l'ampleur à la diffusion de ce qui devient stéréotype. Certains guides, à l'image des Guides bleus, se privent cependant d'illustrations - semble-t-il dans un souci de rigueur et de sérieux. Peut-être leurs auteurs craignaient-ils que les illustrations empêchent de voir: elles imposent d'emblée des images, qui marquent le lecteur au point de l'aveugler au moment de la contemplation réelle de la ville.

\subsection{Partis pris historiques au service de l'idéologie coloniale dans les guides}

Pour raconter la ville coloniale, les auteurs de guides se rangent idéologiquement du côté de la colonisation. Ils ancrent historiquement l'Algérie dans le giron de la France. Le nom du guide est essentiel pour le chercheur: en quête de son objet d'étude, c'est au nom qu'il commence à réfléchir, car c'est ainsi qu'il trouvera les sources recherchées. C'est aussi par le nom que le voyageur qui prépare son départ appréhende d'abord sa destination. Les guides d'Alger ou d'Algérie sont rattachés à la France et à d'autres espaces extérieurs à l'Afrique du Nord. En 1909, le Guide routier et aérien Continental concerne à la fois la France, l'Algérie, la Tunisie et le Nord de l'Espagne $^{13}$. Le Guide Michelin Les Pays du Soleil inclut quant à lui la Corse, la Riviera française, l'Italie, l'Egypte et la Tunisie en plus de l'Algérie ${ }^{14}$. Le fait de rattacher l'Algérie à l'Europe méditerranéenne témoigne, au-delà de l'aspect pratique pour les touristes qui voyagent autour de la Méditerranée, d'une appropriation symbolique évidente. Il est d'ailleurs souvent précisé que concernant la douane, l'Algérie est soumise au même régime que la France. Les guides touristiques prennent part à la construction régionale et nationale : il s'agit d'une thèse défendue par Daniel Nordman, qui évoque le cas de l'Alsace-Lorraine ${ }^{15}$. Par leur nom, les guides participent donc à consolider l'empire colonial, à rattacher l'Algérie à la France.

Outre la toponymie, l'autre parti pris historique essentiel dans les sources est l'idée selon laquelle les Français perpétuent en Algérie l'œuvre de la Rome antique. Les Français y légitime leur retour en se posant en héritiers des Romains. L'argument touristique est évident : le voyage en Italie est une tradition culturelle occidentale depuis plusieurs siècles ${ }^{16}$, et les ruines antiques

\footnotetext{
${ }^{13}$ Guide routier et aérien Continental : France, Algérie, Tunisie et Nord de l'Espagne, Paris, Chaix, 1909.

${ }^{14}$ Guide Michelin Les Pays du Soleil : Riviera française, Corse, Italie, Sicile, Egypte, Algérie, Tunisie, Paris, Michelin, 1912.

${ }^{15}$ NORDMAN, Daniel, Les Guides-Joanne, ancêtres des Guides bleus, in NORA, Pierre, Les Lieux de mémoire, t. II, La Nation, Paris, Gallimard, 1986, pp. 529-567, pp. 554-557.

${ }^{16}$ Sur le voyage des Français en Italie à l'époque moderne voir: BERTRAND, Gilles, Le Grand Tour revisité. Pour une archéologie du tourisme: le voyage des Français en Italie, milieu XVIIIe siècle-début XIe siècle, Rome, Ecole française de Rome, 2008.
} 
présentes en Algérie constituent un atout important pour séduire les voyageurs. Le caractère grandiose des ruines antiques présentes dans les environs d'Alger doit procurer au touriste une émotion toute particulière : à Cherchel, à Tipasa ou au tombeau de la Chrétienne, le touriste se conduit comme en Grèce ou en Italie, et arpente les vestiges manuel d'archéologie à la main. Les traces du passé deviennent un miroir des origines et le tourisme de ruines rend le voyage en Algérie initiatique. La patrimonialisation des vestiges romains d'Alger et de ses environs s'opère au sens étymologique du terme : les Français affichent rapidement leur volonté de préserver les ruines, avec l'idée que cela n'a pas été fait par les « indigènes » durant des « siècles obscurs »- ou du moins envisagés comme tels. Jacques Frémeaux cite à ce sujet Émile-Félix Gautier, un géographe par ailleurs souvent cité dans les guides : «Un historien arabe n'a rien de commun avec Salluste, Tite-Live ou Polybe, voire Procope ; Ceux-ci sont des cerveaux occidentaux, avec qui nous sommes de plain-pied $»^{17}$. Émile-Félix Gautier théorise ainsi l'existence d'un fossé irréductible entre les auteurs anciens desquels il se réclame et les Arabes. Ainsi, la présence romaine en Algérie préfigure la présence française: cette lecture idéologique de l'histoire imprègne quantité de guides touristiques. Les partis pris historiques au service de l'idéologie coloniale posent les bases historiques de l'imaginaire colonial et forgent des représentations par lesquelles Alger est à la fois joyau colonial et destination touristique.

\section{Voyager dans l'empire français : quand le joyau colonial se fait destination touristique}

\subsection{La traversée du lac français}

De Paris à Alger, en passant par Marseille... Le voyage à Alger revêt une signification particulière pour les Français : l'Algérie est proche de l'Europe, intégrée à la « civilisation ». Ce sont les pratiques propres au tourisme moderne qui permettent aux Français de se rendre à Alger, et qui en font une destination privilégiée. Les guides touristiques détaillent avec soin les préparatifs du voyage et les informations nécessaires pour l'arrivée à Alger. Le voyage à Alger est d'abord l'occasion d'une traversée de l'hexagone. Marseille en est une étape essentielle : la cité phocéenne est le port historique du départ vers Alger. D'étape en étape, les distances sont indiquées et les lieux traversés sont décrits : la route fait partie du voyage. Au fil de la période, les traversées se font plus courtes et plus fréquentes, les paquebots plus gros et plus confortables.

\footnotetext{
${ }^{17}$ GAUTIER, Emile-Félix, Le passé de l'Afrique du Nord: les siècles obscurs, Paris, Payot, 1937, p. 64, cité dans FREMEAUX, Jacques, Souvenirs de Rome et présence française au Maghreb : essai d'investigation, in VATIN, JeanClaude (dir), Connaissances du Maghreb. Sciences sociales et colonisation, Paris, Editions CNRS, 1984, pp. 29-46, p. 31.
} 
Dans les années 1930, la traversée s'effectue en vingt heures ${ }^{18}$, et en dix-sept heures à l'aprèsguerre $^{19}$. La liaison aérienne révolutionne le voyage à Alger: "Aujourd'hui, Paris est à deux heures d'Alger par voie aérienne ${ }^{20}$, annoncent les auteurs d'un guide de 1961. Au fil du XX siècle, les modalités du voyage à Alger évoluent de manière spectaculaire, et modifie la signification même du voyage des Français à Alger : plus facile, plus rapide, l'Algérie est plus proche de la France que jamais dans les années qui précèdent l'indépendance.

Pour un Français, l'acte de traverser la Méditerranée n'a rien d'anodin ; la mare nostrum est familière, chargée de nostalgie, au sens le plus étymologique. Loin d'être une frontière, la Méditerranée est un « centre nature ${ }^{21}$ " qui fait le lien entre la France métropolitaine et l'Algérie. Avec la révolution des transports, Alger devient une destination à la mode ; une symbolique de la Méditerranée participe incontestablement de ce phénomène d'engouement touristique autour de la ville coloniale. Selon une comparaison développée par les partisans de l'Algérie française au cours de la guerre d'indépendance, la Mer Méditerranée sépare la France et l'Algérie comme la Seine coupe Paris en deux ${ }^{22}$. Les auteurs des guides développent l'idée qu'Alger n'est qu'un prolongement du Sud de la France. La Méditerranée semble d'abord exister comme un paysage : à ce titre, l'Algérie ressemble à la Provence ou à la Corse. Cette impression de continuum est d'une telle intensité que pour les rédacteurs du Guide Michelin de 1927, « il n'y a pour ainsi dire plus de Méditerranée» ${ }^{23}$. Les guides touristiques, reliés au phénomène plus général de tourisme à Alger, participent de facto d'une construction intellectuelle, d'un système de représentations de l'espace méditerranéen.

La mythification de l'apparition fait d' "Alger la blanche » une image du patrimoine commun, qui rend la ville familière pour tous : ainsi, la première fois à Alger est déjà un retour. La page de description de la baie d'Alger depuis le large est un poncif du guides du corpus : décrite comme une épiphanie, la découverte d'Alger est l'un des événements marquants du voyage. Les topoi de la description du paysage méditerranéen resurgissent immanquablement : le soleil inonde la baie, l'azur du ciel et des eaux se rejoignent. Les termes de «spectacle» ou de «panorama » insistent sur l'attitude contemplative que doit adopter le voyageur au moment où il découvre la baie :

\footnotetext{
${ }^{18}$ Livret-Chaix mensuel, Algérie, Tunisie, Maroc, Paris, Chaix, 1937.

${ }^{19}$ CANTIER, Jacques, s. v. «Politique touristique», in VERDES-LEROUX, Jeannine, L'Algérie et la France, Paris, Robert Laffont, 2009, pp. 701-702.

${ }^{20}$ Algérie Sahara, Guide touristique, commercial, industriel édité au profit des œuvres sociales de l'orphelinat mutualiste des polices de France et d'Outre-mer, Paris, Heintz, 1961, p. 17.

${ }^{21}$ BOURGUET, Marie-Noëlle, Introduction, in BOURGUET, Marie-Noëlle, LEPETIT, Bernard, NORDMAN, Daniel, SINARELLIS, Maroula (dir.), L'invention scientifique de la Méditerranée. Egypte, Morée, Algérie, Paris, Editions EHESS, 1998, p. 28.

${ }^{22}$ THENAULT, Sylvie, L'Algérie assimilée ? Populations et territoires. Du mythe à la réalité, in SARAZIN, Jean-Yves, RAHMANI, Zahia, (dir.), Catalogue de l'exposition « Made in Algeria » au Mucem de Marseille, Vanves, Hazan, 2016, pp. 143-164, p. 143.

${ }^{23}$ Guide Michelin Maroc, Algérie, Tunisie, Paris, Michelin, 1927, p. 2.
} 
On commence à apercevoir la côte d'Algérie environ deux heures avant l'arrivée à quai d'Alger. Il faut se tenir sur le pont pour assister à ce beau spectacle (...). Le panorama que le passager a sous les yeux est de toute beauté. Nulle description ne peut rendre la majesté de ce spectacle $^{24}$.

L'apparition d'Alger est une expérience sensible. Ainsi, l'épique traversée de la Méditerranée est une étape à part entière du voyage à Alger. L'apparition d'Alger n'est rendue possible que par le bateau, le moyen de transport originel du voyage des Français à Alger. Le choix de ne pas enjamber par avion le lac français est récompensé par la contemplation d'un mythe niché au cœur d'une baie.

\subsection{Pour l'Algérie française. La voix colonialiste dans les guides d'Alger et la célébration de la conquête}

«Pour les Français, la circulation est entièrement libre. Il ne faut pas oublier que l'Algérie n'est que le prolongement de la France !» ${ }^{25}$. Ces mots de Félix Falck en 1926 illustrent bien l'idée que les guides touristiques d'Alger sont, de manière générale, pétris d'idéologie colonialiste. Si les processus de tourisme et de colonisation ne sont évidemment pas à mettre sur le même plan, ils sont d'après les historiens Colette Zytnicki et Habib Kazdaghli le produit d'un même contexte : les transports qui acheminent les touristes sont le fruit d'une révolution qui sert aussi, et en premier lieu, à l'armée de la conquête. De même, si le tourisme relève de l'appropriation symbolique d'un territoire, la colonisation se définit quant à elle par une domination violente réalisée par les armes. Dans les deux cas, il s'agit de maîtriser le territoire ${ }^{26}$. Visiter l'Algérie apparaît comme un acte éminemment patriotique aux yeux de l'administration coloniale. Alger est considérée comme la vitrine de l'œuvre française, une propagande vivante de ses réalisations, la démonstration de sa capacité à sauvegarder et à mettre en valeur les richesses historiques et naturelles du pays, mais également comme un moyen de croissance économique.

En 1930, un siècle après la conquête d'Alger, les fêtes du Centenaire ont lieu dans toute l'Algérie. Il s'agit d'un événement patriotique et d'une occasion privilégiée pour les touristes de se rendre à Alger; des programmes des réjouissances sont glissés dans les pages des guides. A cette occasion, le touriste devient l'héritier des gloires de la conquête. Le guide Michelin de 1930

\footnotetext{
${ }^{24}$ Alger en poche. Guide algérien et indicateur des rues d'Alger, Alger, L. Bayard et cie, 1908, p. 4.

${ }^{25}$ FALCK, Félix, op. cit., p. 19.

${ }^{26}$ Introduction, in ZYTNICKI, Colette, KAZDAGHLI, Habib, (dir.), Le tourisme dans l'empire français : politiques, pratiques et imaginaires (XIXe-XXe siècles) : un outil de la domination coloniale ?, Paris, Publications de la Société française d'histoire d'Outre-mer, 2009, p. 7.
} 
accorde une grande importance au Centenaire de la conquête. Un feuillet de deux pages est glissé dans les pages du guide: il décrit le programme des fêtes organisées dans toute l'Algérie à cette occasion. Il s'agit d'une fête politique, au sens de l'historien Alain Corbin : une fête politique a pour fonction, jusqu'à la fin de la III $^{\mathrm{e}}$ République, de produire de la légitimité27. Dans les guides touristiques d'Alger, la voix colonialiste recouvre toutes les autres. En promouvant des événements tels que le Centenaire de la conquête en 1930, les guides participent d'une volonté d'affirmer ou de réaffirmer la grandeur de l'empire français.

\subsection{Au miroir de Paris : l'autre capitale française}

A l'époque coloniale, Alger est une ville française presque comme les autres. Capitale de la colonie devenue ensemble de départements français, l'ancienne place forte de la Régence est forgée à l'image de la ville française. Comme il doit «faire » le voyage à Paris, le touriste doit «faire» le voyage à Alger. En creux, une histoire de la métropole au miroir de la colonie se dessine : il ne s'agit pas d'une histoire comparée du voyage à Paris et du voyage à Alger, mais bien de l'analyse d'un jeu de miroir pour laquelle les guides touristiques sont une source privilégiée.

Le parallèle entre Paris et Alger apparaît explicitement sous la plume des auteurs de guides. La fréquente référence aux arcades de la rue Bab-el-Oued, qui rappellent celles de la rue de Rivoli, plante le décor algérois aux yeux des touristes. La ville qu'ils s'apprêtent à découvrir n'est pas tout à fait l'Afrique du Nord, mais plutôt un prolongement de l'Europe en Afrique.

Ce parallèle entre les deux villes ne se limite pas aux premières années suivant la conquête, comme l'atteste ce passage du guide de Jean du Taillis de 1923 : «Le premier contact avec Alger surprend [...]; on ne comptait pas trouver une capitale si peu province, un pays si français» ${ }^{28}$. Alger n'est pas la province : son rapport avec Paris est tout autre. Le statut de capitale secondaire semble lui être conférée : elle est un autre Paris, sur un autre continent. Il n'est nulle question de concurrence entre les deux villes : l'une est le cœur de l'empire français, l'autre est son reflet sur la rive sud de la Méditerranée. La ville d'Alger représente Paris en Afrique. Comme l'on plante un étendard pour s'approprier un territoire, Alger se dresse en Afrique du Nord au nom de la France.

\footnotetext{
${ }^{27}$ CORBIN, Alain, GERÔME, Noëlle, TARTAKOWSKY, Danielle (dir.), Les usages politiques de la fête aux XIXème et XXème siècles, Paris, Publications de la Sorbonne, p. 9.

${ }^{28}$ TAILLIS, Jean (du), op. cit., p. 57.
} 


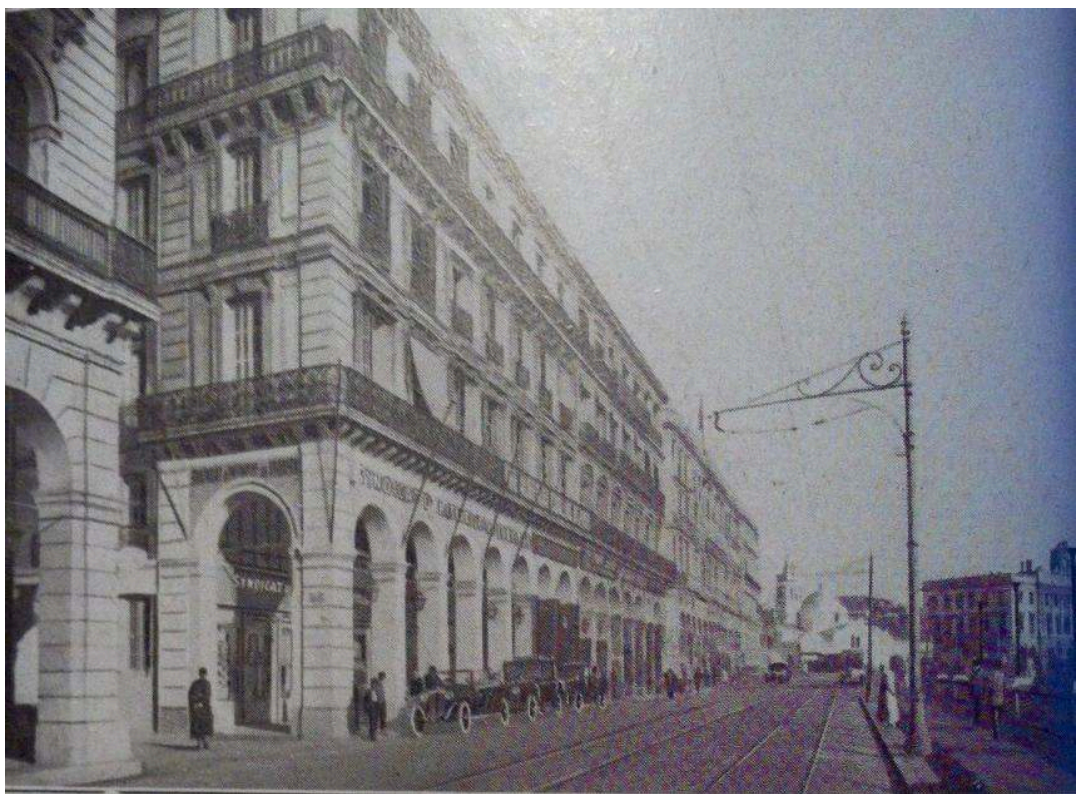

Figure 4. La rue Bab-el-Oued.

[Source : Alger et l'Algérie, livret-guide illustré, publié par le Syndicat d'initiative de tourisme (ancien Comité d'hivernage algérien), Alger, Imprimerie Léon, 1926].

A Alger, les pratiques touristiques sont européennes. La pratique anglaise de l'hivernage, par exemple, contribue à installer durablement le phénomène touristique à Alger. Cependant, celle-ci tend à s'essouffler dès le début du XX ${ }^{\mathrm{e}}$ siècle: la fréquentation des hôtels baisse, et les Anglais se font moins nombreux. Quelques Américains, Allemands et Russes découvrent Alger, sans toutefois initier un tourisme de masse. L'historienne Colette Zytnicki opère d'ailleurs une distinction entre les touristes et les hiverneurs. Selon elle, les seconds appartiennent aux classes supérieures européennes, principalement anglaise, française, allemande, autrichienne ou russe, venues passer les mois d'hiver dans des hôtels de luxe ou des villas prestigieuses. Les touristes, quant à eux, sont des bourgeois moyens qui traversaient la colonie en quelques semaines ${ }^{29}$.

L'hivernage qui avait cours à Alger était intimement lié à la pratique du voyage de santé, qui connaît un essor important jusqu'à la Belle époque. Le soleil et la chaleur deviennent progressivement des objets de convoitise des voyageurs. Même si « l'invention du bronzage » date $\mathrm{du} \mathrm{XX}^{\mathrm{e}}$ siècle selon l'historien Pascal Ory ${ }^{30}$, l'héliotropisme est déjà manifeste au XIX ${ }^{\mathrm{e}}$ siècle. Le soleil méditerranéen est un attrait évident sur lequel les auteurs de guides insistent pour séduire les touristes. Outre le soleil, la qualité de l'air est également une bonne raison de faire le voyage à Alger. Les « promenades hygiéniques» ${ }^{31}$ sont encouragées par les auteurs des guides. L'air algérois réunit les conditions idéales pour des balades pédestres, notamment sur les hauteurs de la ville,

\footnotetext{
${ }^{29}$ ZYTNICKI, Colette, " “Faire l'Algérie agréable ”. Tourisme et colonisation en Algérie des années 1870 à 1962 », in Le Mouvement Social, 242, 1/2013, pp. 97-114, p. 99.

${ }^{30} \mathrm{ORY}$, Pascal, L'invention du bronzage : essai d'une histoire culturelle, Paris, Complexe, 2008.

${ }^{31}$ Guide pratique Conty Algérie et Tunisie, Paris, Chaix, 1904, p. 49.
} 
celles-là mêmes prisées par les hiverneurs. Dans le Guide bleu de 1955, une place est faite aux «formes modernes du tourisme " qui privilégient le plein air: le camping et les auberges de jeunesse. L'auteur Magdelaine Parisot évoque "l'idéal social et moral» ${ }^{32}$ qui gouverne à ces nouvelles pratiques. La localisation des terrains récemment aménagés pour recevoir des campeurs ainsi que l'adresse de la première auberge de jeunesse (rue Sadi-Carnot, au centre de la ville coloniale) sont recensés dans le guide.

En 1908, le guide Alger en poche. Guide algérien et indicateur des rues d'Alger ${ }^{33}$ constitue une source particulièrement intéressante sur les distractions algéroises. Dans une rubrique intitulée « Alger s'amuse ", le guide offre un aperçu des différentes salles de spectacle algéroises de la Belle époque : outre le théâtre municipal, le Kursaal-Nouveau théâtre, le casino music-hall de la rue d'Isly et le Grand cirque franco-américain s'ouvrent désormais aux touristes de passage à Alger. Les trois "cinématographes" de la ville sont également évoqués: l'American-Cosmograph, l'Omnia-Cinéma Pathé et le Royal Cinéma ${ }^{34}$. Ainsi, comme le voyage à Paris, le voyage à Alger est un incontournable pour l'élite française cultivée - les lecteurs de guides, en somme. Mais le tourisme colonial des Français à Alger se singularise du fait de la cohabitation avec une altérité, dominée qui plus est.

\section{Le guide touristique en situation coloniale : l'autre Alger}

\subsection{Des Français à Alger en quête d'Orient}

A Alger, les Français cohabitent avec une population extra-européenne et préexistante à la colonisation, qu'elle qualifie rapidement $d$ ' « indigène ». La ville coloniale est modelée à l'image de Paris et beaucoup d'Européens s'y installent, mais elle reste aux yeux des voyageurs une porte d'entrée vers l'inconnu et l'altérité. Alger a une autre facette: celle d'une ville arabe et « indigène ». Alger, ou l'Orient à une journée de Marseille en bateau... Ainsi pourrait-on résumer un des arguments majeurs qui encouragent les Français à voyager à Alger. Le fond et la forme des guides sont imprégnés de la charge symbolique très forte du voyage en Orient : le goût d'Alger y est intimement lié. Dans la Casbah, "nous sommes en plein Orient» ${ }^{35}$ : le quartier est emblématique de l'Alger précolonial, il s'agit d'un point de passage obligatoire de tous les itinéraires touristiques. Cœur arabe de la cité, elle cristallise l'ensemble des topoi relayés par les guides d'Alger. Elle est souvent décrite comme un «amusant labyrinthe », où le voyageur est

\footnotetext{
${ }^{32}$ PARISOT, Magdelaine, Guide bleu Algérie-Tunisie, Paris, Hachette, 1955, pp. XCVI-XCVII.

${ }^{33}$ Alger en poche. Guide algérien et indicateur des rues d'Alger. Alger, L. Bayart et cie, 1908.

${ }^{34}$ Ibidem, p. 12.

${ }^{35}$ Alger en poche. Algiers pocket edition, Alger, Soubiron, 1930, p. 11.
} 
confronté au " mystère » et à l" " aventure " ${ }^{36}$. Ainsi, Alger est pour les touristes le réceptacle de représentations sur l'Orient. Un Orient pourtant altéré par la colonisation : «Il serait impardonnable de venir à Alger sans avoir vu la Kasbah - ou ce qu'il en reste, la pioche impitoyable des démolisseurs civilisés ayant détruit les plus beaux coins de la ville orientale» ${ }^{37}$. En territoire colonisé, l'authenticité de l'Orient est remise en cause par les auteurs de guides ${ }^{38}$. Ils sont nombreux à condamner ce que les Français font subir au vieil Alger depuis la conquête. Ces critiques sont paradoxales, car elles cohabitent avec une célébration de l'Algérie française et un parallèle élogieux établi entre Alger et Paris.

En plus de la situation coloniale qui redouble la signification de cette altération de l'Alger précolonial, la tension qui distingue les définitions du touriste et du voyageur se fait sentir. Le touriste est le voyageur sans aventures, qui déambule dans un monde fini et standardisé, modelé selon les goûts et les commodités occidentales. En effet, l'empire colonial n'est pas le champ privilégié de la rêverie d'aventure, selon l'historien Sylvain Venayre ${ }^{39}$. Il apparaît que la critique des villes coloniales européanisées pourrait entrer en correspondance avec cette «mystique moderne de l'aventure» ${ }^{40}$ qui désavoue le tourisme. Rien n'est plus authentique, rien n'est plus vierge en territoire conquis par les touristes... Cela peut surprendre le lecteur contemporain, dans la mesure où les guides sont les manuels du touriste par excellence. Le thème d'un Orient domestiqué et encadré par la présence coloniale rend par ailleurs Alger rassurante aux yeux des auteurs et des touristes. En somme, la destination Alger synthétise exotisme et confort, orient et maîtrise. Définitivement, Alger représente aux yeux de beaucoup de voyageurs l'Orient proche et accessible.

\subsection{Le passé précolonial, les hommes et les femmes «indigènes » : entre mise à} distance et fantasmes

Le passé de l'Algérie avant la conquête est l'objet d'un discours ambivalent : tantôt assimilé à des temps obscurs, tantôt patrimonialisé et regretté, ce passé précolonial n'occupe pas une place prépondérante dans les guides. Archaïser la société colonisée et son passé pour mieux glorifier la modernité des colonisateurs et légitimer l'empire : il semble que ce principe ait été appliqué par nombre d'auteurs de guides, en accord avec l'idéologie dominante. Par passé précolonial, nous

\footnotetext{
${ }^{36}$ Le Petit guide d'Alger, Alger, J. Le Bourgeois, 1909, p. 5.

${ }^{37}$ Guide pratique Conty Algérie et Tunisie, Paris, Chaix, 1904, p. 45.

${ }^{38}$ Sur la question du statut de la Casbah, de ses transformations et de sa patrimonialisation par le pouvoir colonial, voir: OULEBSIR, Nabila, Les usages du patrimoine. Monuments, musées et politique coloniale en Algérie, 1830-1930, Paris, Éditions de la Maison des sciences de l'homme, 2004.

${ }^{39}$ VENAYRE, Sylvain, La Gloire de l'aventure, Genèse d'une mystique moderne, 1850-1950, Paris, Aubier, 2002 , p. 89.

${ }^{40}$ Ibidem.
} 
entendons ici le passé non-occidental d'Alger, à l'exclusion de la période romaine. Si les auteurs de guides peuvent mettre en valeur les vestiges de ce passé de temps à autres, ils l'aplanissent et relayent une image stéréotypée et immobile du vieil Alger. Dans les pages qui concernent l'histoire de la ville, le déséquilibre est flagrant entre la période romaine-carthaginoise et la période arabe-turque. Entre le glorieux passé antique et la colonisation française apparaît ainsi un trou béant, que l'on pourrait nommer « les temps obscurs » de l'Algérie.

Les contacts entre voyageurs français et « indigènes » sont de fait limités et le touriste ne les fréquente pas, en dehors peut-être des cicérones. Les publicités le suggèrent : les hôtels et les commerces sont tenus par les Européens. Les « indigènes " ne sont cependant pas tout à fait absents des guides. Dans une veine indigénophile, un hommage est presque unanimement rendu par les auteurs des guides d'Alger aux combattants "indigènes" morts pour la France. Dès l'entre-deux-guerres, Félix Falck leur consacre quelques lignes au ton épique :

\begin{abstract}
Aujourd'hui l'Algérie, merveilleusement prospère, est française autant que nos vieilles provinces. La guerre de 1914-1918 a prouvé de façon éclatante son loyalisme. Les plus lointaines, donc les moins assimilables de ses tribus, restèrent sourdes aux conseils d'une propagande qui ne recula cependant devant aucun moyen et ses fils vinrent nombreux verser leur sang sur les champs de bataille de France et de Belgique ${ }^{41}$.
\end{abstract}

Cette saillie indigénophile se justifie chez Félix Falck par la loyauté et le dévouement des troupes « indigènes » durant la Grande Guerre. La reconnaissance vis-à-vis des "indigènes » prend donc part à un discours fréquemment employé par les auteurs de guides du $\mathrm{XX}^{\mathrm{e}}$ siècle.

Très souvent présentes sur les illustrations des guides, les femmes algéroises font l'objet de fantasmes multiples et toujours implicites, car peu avouables d'un point de vue moral. Alger est souvent personnifiée: elle est une femme séduisante et voluptueuse que les Français sont parvenus à conquérir. En écho aux stéréotypes traditionnels de la femme orientale, les guides touristiques livrent des représentations peu variées des femmes d'Alger. Cette fascination se mêle parfois à la mise en garde aux voyageurs :

Lorsque que nous les regardons du haut de nos terrasses, jeunes ou vieilles ont toujours quelque chose à faire pour traverser leurs cours et y passer et repasser sous nos yeux (...). Du reste, ne vous y frottez pas de trop près; presque toutes ont la gale, qui leur est donnée par leurs enfants ${ }^{42}$.

\footnotetext{
${ }^{41}$ FALCK, Félix, op.cit., p. 9.

${ }^{42}$ CHARBONNEAU, Jean-Eugène, A la découverte de l'Afrique du Nord : ce qu'il faut voir, savoir et lire... , Bourg-enBresse, Editions touristiques et littéraires, 1951, p. 127.
} 
L'argument sanitaire est ici invoqué pour déconseiller aux voyageurs les rapprochements avec les femmes "indigènes", qui feraient tout pour attirer le regard des voyageurs. La peur des maladies vénériennes - que l'auteur ne cite pas, probablement pour des raisons morales - peut aussi justifier cet avertissement.

Le fantasme d'une sexualité libre et exotique est une composante de l'attrait exercé par l'Algérie aux yeux de certains voyageurs. Dans l'Algérie coloniale, la prostitution était une institution avec son système juridique et ses espaces urbains. A Alger, la prostitution était concentrée dans la haute Casbah. En 1937, la ville compte trente-sept maisons de tolérance selon l'historienne Barkahoum Ferhati : la Casbah était alors une des attractions touristiques les plus prisées d'Alger, une des raisons pour lesquelles la loi Marthe Richard (qui impose la fermeture des maisons closes françaises en 1946) ne fut pas appliquée à Alger ${ }^{43}$. Pour l'historienne Christelle Taraud qui a consacré sa thèse à la prostitution coloniale, la Casbah de Tunis était «l'égout séminal de la nouvelle ville européenne» ${ }^{44}$. Il nous semble que l'expression peut également être appliquée à la Casbah d'Alger. Christelle Taraud parle d'une violence sexuelle structurelle, qui repose sur une politique d'exception, l'indigénat.

Si l'écrasante majorité des guides du corpus étudié pour cette recherche ne font pas mention de la prostitution, des guides spécifiques existent. Le Guide rose est un exemple significatif: l'ouvrage consistait en un annuaire des maisons closes. En 1934, le Guide rose fait mention de onze maisons de tolérance dans la basse Casbah ${ }^{45}$. Il n'existait pas d'édition spécifique pour l'Algérie : chaque annuaire était consacré à Paris, à la province et aux colonies. S'il n'est pas uniquement destiné aux voyageurs de passage à Alger, ce guide clandestin reprend les codes des guides traditionnels. Alors que le Guide bleu hérite de la vision patriotique et conservatrice du GuideJoanne, le Guide rose est celui des maisons closes de la France et de son empire colonial...

\footnotetext{
${ }^{43}$ FERHATI, Barkahoum, s. v. « Maisons de tolérance de la Casbah d'Alger », VERDES-LEROUX, Jeannine, op. cit., pp. 547-548.

${ }^{44}$ TARAUD, Christelle, La prostitution coloniale, Algérie Maroc Tunisie (1830-1962), Paris, Payot, 2003, p. 12.

${ }^{45}$ Le Guide rose. Annuaire indicateur des maisons et salons de société, maisons de massage et de rendez-vous de Paris, province et colonies, Paris, Annuaire GTC, 1936.
} 


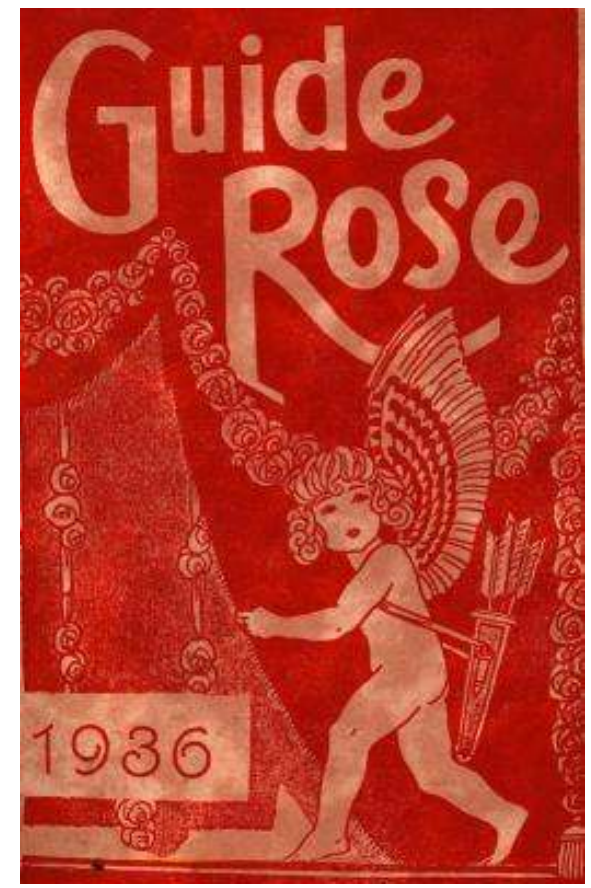

Figure 5. Couverture du Guide Rose.

[Source : Guide rose. Annuaire indicateur des maisons et salons de société, maisons de massage et de rendez-vous de Paris, province et colonies, Paris, Annuaire GTC, 1936].

Le Guide rose disparaît au moment de la loi Marthe Richard en 1946. Le voyage dans "la voluptueuse Alger, parée de toutes ses séductions» ${ }^{46}$ nourrit à plus d'un titre les représentations liées aux femmes de la population colonisée.

\subsection{Ne pas tout dire. L'absence et la violence dans les guides d'Alger}

Les guides d'Alger ne sont pas les seuls à exclure de leurs pages les habitants des villes. L'aveuglement sur les conditions misérables dans lesquelles vivent la plupart des « indigènes » est manifeste, mais n'est pas uniquement lié au contexte colonial: le voyage d'agrément et la confrontation à la misère locale n'ont jamais fait bon ménage. Pourtant, la situation coloniale et la violence qu'elle entraîne pour les populations colonisées ne sont pas sans conséquence pour le tourisme, donc pour le contenu des guides de voyage. La violence des propos de certains auteurs confirme que les «indigènes » sont dépeints de manière extrêmement péjorative dans les guides. Les hommes pauvres, le plus souvent « indigènes » mais aussi juifs, sont les cibles privilégiées de ces invectives. Les plus démunis parmi la population locale sont les avatars d'une civilisation dégradée :

\footnotetext{
${ }^{46}$ TAILLIS, Jean (du), op. cit., p. 55.
} 
En face de l'arrêt de tramway est un terrain vague où, le dimanche matin, tous les débris lamentables de la civilisation sont mis en vente. Voir en face le barbier arabe, en train de raser, en plein vent, le cuir chevelu de ses coreligionnaires, en se servant d'instruments moyenâgeux et rouillés [...]. On a de la peine à concevoir qu'il puisse exister des êtres humains assez indifférents aux agréments de la propreté pour y élire domicile. Passons! ${ }^{47}$.

La dureté des mots que Gubb emploie ici s'inscrit ainsi dans une certaine continuité : dans les guides, soit la misère est dissimulée, soit elle est moralement réprouvée.

Le guide touristique repose sur une conscience de soi et une conscience des autres, et fabrique ou diffuse des idées reçues et des stéréotypes. Dans l'Alger coloniale, la conscience de l'altérité amène souvent à des préjugés racistes et le sentiment d'une culture dominante, celle du colonisateur français. Le discours est prégnant dans ce passage du Guide Michelin de 1927 :

\begin{abstract}
Un trait domine l'histoire des peuples d'Afrique du Nord : livrés à eux-mêmes, ils n'ont jamais connu ni paix, ni unité, ni prospérité ; ils n'ont joui de bienfaits qu'à deux moments, placés presque aux deux pôles de leur destinée : celui de l'occupation romaine et celui, qu'ils vivent encore, de l'occupation française ${ }^{48}$.
\end{abstract}

Ces lignes prouvent qu'un ordre social vertueux pour les colonisateurs et les colonisés continuent à être légitimé. L'hégémonie de ce type de discours est total dans les guides d'Alger : aucun auteur ne s'élève pour dénoncer la colonisation, y compris à la fin de la période. La domination coloniale transparaît parfois violemment dans les guides d'Alger.

«Le but est de nier la chose. La nommer le moins possible, lui donner le sens de son propre contraire $^{49}$ ». Cette citation de Roland Barthes peut s'appliquer au traitement réservé à la guerre dans les guides d'Alger. Leur silence à ce sujet est frappant, même si les éditions se font plus rares entre 1954 et 1962. Le plus souvent, les auteurs se contentent de réaffirmer avec véhémence leur attachement à l'Algérie française. Pourtant, avant la guerre d'Algérie, d'autres guerres ont pu êtres évoquées dans les guides, notamment la Première Guerre mondiale. Cette dernière fait l'objet de quelques remarques dans les guides, toujours reliées à des considérations touristiques. L'ombre portée de la guerre atteint durement le tourisme, et il apparaît qu'aucun guide d'Alger n'est publié en France entre 1914 et 1918. Comme pour la Première guerre mondiale, l'absence de guides pendant la période 1939-1945 est manifeste - à notre connaissance, le premier guide d'Alger d'après-guerre ne date que de $1950^{50}$. Cependant, dans tous les guides postérieurs, nulle

\footnotetext{
${ }^{47}$ GUBB, Alfred-Samuel, op. cit., pp. 15-17.

${ }^{48}$ Guide Michelin Maroc, Algérie, Tunisie, Paris, Michelin, 1927, p. 11.

${ }^{49}$ BARTHES, Roland, Grammaire africaine, in ID., Mythologies, Paris, Seuil, 1957, p. 130.

${ }^{50}$ RICARD, Prosper, Guide bleu Algérie Tunisie, Paris, Hachette, 1950.
} 
mention de la Seconde guerre mondiale, ou même du rôle d'Alger pendant celle-ci, qui a pourtant été essentiel.

Dans ces circonstances, le silence sur la guerre d'Algérie est assourdissant, mais peu étonnant. Le premier guide paru après le début de la guerre est le Guide bleu de 1955, dirigé par Madgelaine Parisot $^{51}$. Aucune mention des « événements » n'est lisible dans ces pages. La première référence à la guerre d'Algérie contenue dans un guide se trouve dans l'éditorial du Guide Michelin AlgérieSahara de 1956. «Confiants dans l'avenir de l'Algérie et malgré les récents événements d'Afrique du Nord, les Services de Tourisme Michelin ont déjà édité le Guide Maroc-Algérie, hôtels et restaurants $»^{52}$. Ce passage est extrait de l'éditorial du guide, intitulé « l'Algérie France nouvelle ». L'expression mentionnant les "récents événements » est extrêmement allusive, noyée dans une annonce publicitaire pour l'éditeur. Les lignes qui suivent consistent essentiellement en la glorification de l'œuvre française en Algérie. Tous les thèmes de légitimation de la colonisation sont présents: la pacification, la lutte contre l'anarchie, l'instruction, l'hygiène ou encore l'amélioration de l'économie. Ce guide inaugure ainsi la série de guides touristiques qui, jusqu'en 1961-1962, réaffirme avec véhémence la grandeur de l’Algérie française.

Le guide le plus récent du corpus date de $1961^{53}$. Édité à Paris, ses auteurs sont anonymes. Aucune allusion à la guerre ni à de quelconques événements n'est formulée. Les auteurs se contentent de réaffirmer avec force leur attachement à l'Algérie française : "Dès le début, en présence d'une situation anarchique, vide de réalités constructives, de pouvoir créateur, la France, conforme à son génie, s'est trouvée dans l'obligation de bâtir un monde nouveau ${ }^{54}$. Le silence assourdissant des guides sur la guerre d'Algérie est extraordinairement tendu, mais reste tout de même un silence; en 1962, lorsque l'Algérie devient algérienne, les guides de l'Algérie française se taisent définitivement. En 1962, la scission avec la France se concrétise par l'indépendance de l'Algérie, après des années de violence extrême, dont une infime partie seulement a filtré dans les guides de voyage.

\footnotetext{
${ }^{51}$ PARISOT, Magdelaine, Guide bleu Algérie-Tunisie, Paris, Hachette, 1955.

${ }^{52}$ Guide Michelin Algérie Sahara, Paris, Michelin, 1956, p. 1.

${ }^{53}$ Algérie Sahara, Guide touristique, commercial, industriel, cit., 1961.

${ }^{54}$ Ibidem, p. 5.
} 


\section{Conclusion}

«A l'échelle collective, le voyage est volontiers considéré comme une rupture dans l'ordre des choses ${ }^{55}$ : cette affirmation de Sylvain Venayre s'applique au voyage à Alger, tant il a revêtu une importance symbolique aux yeux des Français et nourri leurs représentations de la ville. Joyau colonial, destination touristique de prestige ou véritable lieu de pèlerinage politique, une image résolument singulière et multiple d'Alger est donnée dans les guides touristiques. Les guides de l'Alger coloniale disparaissent totalement en 1962. Une quinzaine d'années s'est écoulée après l'indépendance pour qu'un nouveau guide d'Alger reparaisse en France, en $1977^{56}$.

Un sentiment de défiance vis-à-vis des guides touristiques peut naître chez le lecteur. Sur Alger, démonstration est faite que les représentations du territoire d'un point de vue français sont éminemment politiques. D'une certaine manière, les guides s'imposent comme des filtres empêchant de voir le monde, de le découvrir sans préjugés. Comme le suggère Jean-Eugène Charbonneau, l'ultime solution serait peut-être de s'en débarrasser :

Lecteur, je t'ai trop parlé de Gide pour ne pas finir en te disant comme lui à son Nathanaël : "Et maintenant jette ton livre". Jette tous les livres, et les guides en premier. Fie-toi à ton instinct, à ton propre goût de la découverte ${ }^{57}$.

\footnotetext{
${ }^{55}$ VENAYRE Sylvain, s. v. «Voyage ", in GAUVARD, Claude, SIRINELLI Jean-François (sous la dir. de), Dictionnaire de l'historien, Paris, Presses Universitaires de France, 2015, p. 749.

${ }^{56}$ MODOT, Jean, Guide bleu Algérie, Paris, Hachette, 1977.

${ }^{57}$ CHARBONNEAU, Jean-Eugène, op. cit., p. 260.
} 


\section{L'AUTEUR}

Sihem BELLA est agrégée d'histoire et diplômée de Sciences Po (Paris) en histoire contemporaine. Ses recherches portent sur l'histoire culturelle de l'Algérie coloniale, plus spécifiquement l'histoire du voyage et du tourisme.

URL: < http://www.studistorici.com/progett/autori/\#Bella > 